\title{
Simulação da qualidade da água para suporte em modelos de otimização para planejamento e gestão de recursos hídricos
}

\author{
Water quality simulation to support optimization models \\ for water resources planning and management
}

Ana Paula Dalcin, Guilherme Fernandes Marques

Instituto de Pesquisas Hidráulicas (IPH), Universidade Federal do Rio Grande do Sul (UFRGS), Porto Alegre, RS, Brasil. E-mail: ana.dalcin@ufrgs.br; guilherme.marques@ufrgs.br

\begin{abstract}
Como citar: DALCIN, A. P.; MARQUES, G. F. Simulação da qualidade da água para suporte em modelos de otimização para planejamento e gestão de recursos hídricos. Revista de Gestão de Água da América Latina, v. 16, e15, 2019. https://dx.doi.org/10.21168/rega.v16e15.
\end{abstract}

Resumo Plataformas de simulação da qualidade de água muitas vezes apresentam limitações para responder perguntas sobre a melhor estratégia de intervenção quando o sistema modelado possui múltiplos objetivos e usuários, tornando necessário o emprego de uma ferramenta de otimização. Entretanto, incorporar a representação de processos de qualidade da água em modelos de otimização pode requer simplificações para superar dificuldades computacionais, sem comprometer a efetividade do modelo. Este trabalho tem por objetivo avaliar o emprego de um modelo simplificado para simulação da qualidade da água na Bacia do Rio dos Sinos (RS), possibilitando o seu uso como parte integrante de modelos hidroeconômicos e suporte ao planejamento e gestão de recursos hídricos. Apesar da proposta utilizar um número limitado de divisão de trechos de rio, os resultados mostram que o modelo simplificado conseguiu acompanhar as variações de concentração para os parâmetros Demanda Bioquímica de Oxigênio e Coliformes Termotolerantes, porém com deslocamento da sua posição no espaço (comprimento do rio). Dentro de suas limitações e possibilidades de ajustes, o modelo é de fácil aplicação em rotinas de programação diversas que utilizam técnicas de otimização.

Palavras-chave: qualidade da água, otimização, simulação

Abstract Water quality simulation platforms often have limitations in answering questions about the best intervention strategy when the modeled system has multiple objectives and users, which requires the application of optimization tools. However, incorporating water quality processes in optimization models may demand simplifications to overcome computational barriers without compromising the model effectiveness. This paper aims to analyze the application of a simplified water quality simulation model in the Sinos River Basin (RS), allowing its integration to water resources managing and planning approaches and hydroeconomic models. Although using a limited river reaches division, the results show that the simplified model was able to represent the main concentration variations of the parameters Biochemical Oxygen Demand and Thermotolerant Coliforms but with displacement of its position in the space (river length). Within its limitations and possibilities of adjustments, the model is easy to apply in various programming routines that use optimization techniques.

Keywords: water quality, optimization, simulation

\section{INTRODUÇÃo}

A gestão de recursos hídricos é caracterizada pela natureza multidisciplinar, o que requer o emprego de diferentes (e avanço de novos) métodos para integrar aspectos técnicos, econômicos, ambientais, sociais e legais em uma abordagem coerente. Modelos hidrológicos e de qualidade da água geralmente utilizam técnicas de simulação, nos permitindo avaliar cenários e responder à pergunta "e se?" decorrentes de uma estratégia de intervenção, condição hidrológica, ou combinação de ambas. Embora capazes de representar em detalhe diversos processos, abordagens de simulação apresentam limitações para responder uma outra pergunta: “o que é melhor?”. Responder a essa pergunta requer

Recebido: 25/8/2019 Revisado: 30/12/2019 Aceito: 31/12/2019 
a simulação de várias combinações de cenários diferentes, para comparação dos resultados, o que é viável e eficiente se o sistema modelado for relativamente simples em termos de objetivos, tipos de usuários, preferências dos mesmos e alternativas de intervenção (Loucks \& van Beek, 2017). Entretanto, em sistemas mais complexos, o maior número de objetivos, usuários e alternativas irá invariavelmente resultar em um grande número de cenários, tornando o emprego exclusivo da simulação pouco eficiente. Em alguns desses casos, modelos de otimização podem ser empregados para identificar uma, ou mais, soluções promissoras, sendo posteriormente refinadas com o modelo de simulação (Loucks \& van Beek, 2017).

Entretanto, para incorporar a simulação de processos hidrológicos ou de qualidade da água em modelos de otimização são necessárias simplificações, porém sem comprometer a efetividade do modelo. Dessa forma, para atingir um nível de integração que permita um fluxo interativo e efetivo de informações entre simulação e otimização, diversas barreiras precisam ser superadas (Mckinney et al., 1999). A avaliação da alocação de água em uma bacia hidrográfica, por exemplo, além dos aspectos econômicos relacionados ao seu uso ou escassez, pode estar condicionada a restrições ambientais (disponibilidade hídrica e/ou qualidade), o que faz necessária a avaliação conjunta de modelos econômicos e modelos hidrológicos ou de qualidade da água para simular os possíveis impactos decorrentes do processo de alocação.

Dentro da abordagem da modelagem hidroeconômica, as plataformas de simulação da qualidade de água mais sofisticadas (como a exemplo dos modelos QUAL-2E, HEC-RAS, entre outros) são comumente empregados com o objetivo de avaliar os impactos de alocações de água posteriormente ao processo de otimização. Em Azevedo et al. (2000), o modelo QUAL-2E-UNCAS foi aplicado para simular a qualidade da água resultante do modelo de otimização de alocação de água MODSIM. Contudo, softwares de simulação muitas vezes apresentam dificuldade de serem empregados em análises integradas por serem computacionalmente dispendiosos. Uma única simulação pode levar minutos, horas e até mesmo dias, o que limita o emprego de técnicas de otimização de forma simultânea. Uma abordagem alternativa envolve a criação de modelos que imitam o comportamento por funções aproximadas de maneira a substituir simulações individuais, citando-se, como exemplos, redes neurais artificiais, máquinas de suporte vetorial, entre outros algoritmos de aprendizagem (Bouhlel et al., 2019; Yang et al., 2014; Qian et al., 2006). Entretanto, muitas vezes a sua criação também é dispendiosa devido à quantidade de dados necessários para os processos de treinamento e teste dos algoritmos.

Outra alternativa envolve a substituição de simulações detalhadas por simulações aproximadas através da simplificação da representação do sistema pela exclusão de certas variáveis, processos ou redução da resolução numérica, sendo a precisão sacrificada para reduzir o tempo computacional (Qian et al., 2006). Cai et al. (2003) desenvolveram um modelo hidrológico-agronômico-econômico integrado com o objetivo de alocar água para diferentes usuários, maximizando o benefício econômico. Equações de balanço de massa na rede da bacia hidrográfica e zonas de raízes dos cultivos foram utilizadas para simular a qualidade da água e gerar penalidades de acordo com a concentração de salinidade efluente. A partir da fundamentação teórica do modelo de Cai et al. (2003), Moraes et al. $(2008,2010)$ desenvolveram um modelo econômico hidrológico integrado para identificar a alocação ótima de água entre os diversos usuários da bacia hidrográfica do Rio Pirapama no estado de Pernambuco. Por apresentar problemas de poluição em diversos trechos relacionados à fertirrigação das áreas de cana, o modelo também incorporou a avaliação de qualidade da água através dos parâmetros Demanda Bioquímica de Oxigênio (DBO) e Oxigênio Dissolvido (OD) por meio do emprego de equações do modelo de Streeter-Phelps (Streeter \& Phelps, 1925). O modelo foi ainda empregado na avaliação de instrumentos econômicos alocativos, como a outorga controlada e cobrança pelo uso da água (Moraes et al., 2009).

Bandeira \& Mendes (2009) desenvolveram um modelo matemático de otimização da produção de minério de carvão e internalização dos custos de tratamento do efluente gerado através do emprego de equações de balanço massa para simulação da qualidade da água. Amorim Filho (2013) desenvolveu um modelo hidroeconômico para a bacia do rio Tapacura em Pernambuco, o qual é capaz de determinar a alocação econômica ótima de água para cenários presente e futuro, respeitando restrições de quantidade e qualidade, através da simulação do parâmetro fósforo por equações de balanço de massa e decaimento. Molinos-Senante etal. (2014) propuseram um modelo de otimização para alocação de água que determina o volume de água a ser fornecido para cada usuário, com base em maximização do benefício, e identifica de que fonte o fornecimento deve ser realizado com base em requerimentos de qualidade. Davidsen etal. (2015) propuseram um modelo de otimização que permite comparar os impactos econômicos do cumprimento de vários graus de qualidade de água, considerando descarga de efluentes 
e tratamento de água, através do emprego da equação de Streeter-Phelps para calcular as concentrações de oxigênio dissolvido. Ainda, Wang et al. (2019) utilizaram um modelo de simulação de qualidade da água baseado em equações de balanço de massa e depuração para simular a qualidade da água resultante da otimização da alocação da água de diferentes fontes para diferentes usuários.

Dentro deste contexto, este trabalho tem por objetivo avaliar o desempenho de um modelo simplificado para simulação da qualidade da água na Bacia do Rio dos Sinos (RS), Brasil, possibilitando seu emprego junto a modelos hidroeconômicos no suporte ao planejamento e gestão de recursos hídricos. A abordagem e resultados aqui apresentados foram empregados satisfatoriamente em um modelo de otimização de alocação dinâmica de outorgas (Dalcin, 2019), permitindo a integração de decisões de retirada de água, lançamento de efluentes, enquadramento e investimento em tratamento de esgoto em um único modelo de planejamento de longo prazo.

A fim de avaliar a adequabilidade do modelo de simulação proposto, apresentamos a fundamentação teórica utilizada, seguida da descrição geral do modelo, sua discretização e hipóteses simplificadoras. Em seguida, apresentamos os resultados das simulações para os parâmetros Demanda Bioquímica de Oxigênio e Coliformes Termotolerantes realizadas com o modelo simplificado e com o software HEC-RAS, utilizado como modelo de referência para obtenção de resultados e comparação. 0 modelo de referência foi calibrado a partir de dados de topobatimetria, imagens de satélite e qualidade da água da rede de monitoramento do Rio dos Sinos. A modelagem em HEC-RAS também foi utilizada com o objetivo de avaliar as características hidráulicas do escoamento, tais como, velocidade e tempo de percurso, possibilitando a incorporação destes parâmetros nas equações do modelo simplificado.

\section{METODOLOGIA}

\subsection{Descrição do Modelo Simplificado proposto}

O modelo simplificado foi desenvolvido com base na Lei da Conservação, considerando fluxo unidimensional em x e mecanismos de transporte de massa. A Lei da Conservação estabelece que uma propriedade conservativa (energia, massa e momento) não pode ser criada ou destruída, apenas transferida ou transformada (Martin \& McCutcheon, 1999). Em termos quantitativos, este princípio pode ser expresso como uma equação de balanço de massa para um volume de controle finito englobando todas as transferências de matérias através das fronteiras do sistema e todas as transformações ocorridas dentro do sistema (1) (Chapra, 2008).

Acumulado $=$ transporte \pm cargas externas \pm reações

A advecção e a difusão são mecanismos responsáveis pelo transporte de massa. A advecção é resultante do fluxo unidirecional bem definido e geralmente representa o processo de transporte primário de poluentes em rios e estuários na direção longitudinal (Ji, 2017). Já, a difusão é dividida em duas categorias: molecular e turbulenta. A difusão molecular se refere ao movimento aleatório da massa devido à existência de uma diferença de concentração, enquanto que a difusão turbulenta é o movimento aleatório proveniente de um fluxo turbulento (Martin \& McCutcheon, 1999). Considerando um fluxo unidimensional em x, ao incorporar esses mecanismos na equação da conservação de massa (1), a mesma resulta na equação (2).

$\frac{\partial \mathrm{C}}{\partial \mathrm{t}}=-\mathrm{u} \cdot \frac{\partial \mathrm{C}}{\partial \mathrm{x}}+\mathrm{D} \cdot \frac{\partial^{2} \mathrm{C}}{\partial \mathrm{x}^{2}} \pm$ cargas externas \pm reações

onde: $\mathrm{C}=$ concentração da substância $\left(\mathrm{M} \mathrm{L}^{-3}\right) ; \mathrm{t}=\operatorname{tempo}(\mathrm{T}) ; \mathrm{u}=\operatorname{velocidade}\left(\mathrm{L}^{2} \mathrm{~T}^{-1}\right)$; $\mathrm{D}=$ coeficiente de difusão $\left(\mathrm{L}^{2} \mathrm{~T}^{-1}\right)$.

Ao englobar as cargas externas que entram e saem do sistema, representadas por tributários e fluxos laterais, tais como lançamento de efluentes e captações de água, a equação de conservação de massa pode ser escrita conforme (3).

$\frac{\partial \mathrm{C}}{\partial \mathrm{t}}=\frac{\mathrm{W}_{\text {in }}}{\mathrm{V}}-\frac{\mathrm{W}_{\text {out }}}{\mathrm{V}}-$ u. $\frac{\partial \mathrm{C}}{\partial \mathrm{x}}+\mathrm{D} \cdot \frac{\partial^{2} \mathrm{C}}{\partial \mathrm{x}^{2}} \pm$ reações 
onde: $\mathrm{W}_{\text {in }}=$ carga da substância que entra no sistema $\left(\mathrm{M} \mathrm{T}^{-1}\right) ; \mathrm{W}_{\text {out }}=$ carga da substância que sai do sistema $\left(\mathrm{M} \mathrm{T}^{-1}\right)$.

Por fim, uma substância ainda pode sofrer reações químicas e bioquímicas transformando-se em outros componentes, bem como pode sofrer mecanismos físicos de sedimentação e volatilização. As cinéticas das reações não conservativas podem ser expressas quantitativamente pela lei de ação das massas, a qual coloca que a taxa de variação da concentração é proporcional à concentração dos reagentes (Chapra, 2008). Considerando apenas um reagente, a equação da reação pode ser representada conforme (4).

$\frac{\mathrm{dC}}{\mathrm{dt}}=-\mathrm{k} \cdot \mathrm{C}^{\mathrm{n}}$

onde: $\mathrm{n}=$ ordem; $\mathrm{k}=$ coeficiente de decaimento $\left(\mathrm{T}^{-1}\right)$.

Reações de ordem $n=0,1$ e 2 são as mais comumente empregadas em águas naturais, sendo os parâmetros de qualidade, Demanda Bioquímica de Oxigênio (DBO) e patógenos (coliformes termotolerantes), por exemplo, representados por reações de primeira ordem. Resolvendo (4) analiticamente para $\mathrm{n}=1$, é possível obter a concentração no final do tempo $t$ simulado (5).

$\mathrm{C}=\mathrm{Co}^{-\mathrm{e}^{-\mathrm{k} . \mathrm{t}}}$

onde: $C o=$ concentração inicial da substância em $t=0\left(\mathrm{M} \mathrm{L}^{-3}\right)$.

0 coeficiente de decaimento da DBO ( $\mathrm{K}_{\mathrm{DBO}}$ ) é composto por duas parcelas, uma referente à taxa de decomposição $\left(\mathrm{k}_{\mathrm{d}}\right)$ e outra à taxa de sedimentação $\left(\mathrm{k}_{\mathrm{s}}\right)$. 0 coeficiente de decaimento para patógenos $\left(\mathrm{k}_{\mathrm{col}}\right)$ é, por sua vez, composto por três parcelas, mortalidade, perda devido à radiação solar e perda por sedimentação (Chapra, 2008). De acordo com Chin (2013) os valores de $k_{d}$ podem variar de 0,05 (rio não poluído) a 0,7 (efluentes não tratados), enquanto que para $\mathrm{k}_{c o l}$ a variação situa-se na faixa de 0,8 a 5,5 (Thomann \& Mueller, 1987).

Com base na fundamentação teórica apresentada, o Modelo Simplificado proposto apresenta algumas hipóteses, a fim de facilitar o seu emprego em rotinas de programação e consequente uso em ferramentas de suporte ao planejamento e gestão. A primeira delas considera que o mecanismo de transporte por difusão/dispersão em escoamento em rios tem uma representatividade muito inferior se comparado com a advecção, sendo, portanto, excluído do equacionamento. A segunda consideração é em referência à avaliação do escoamento apenas em regime permanente (estacionário). Ou seja, é considerado que a massa se mantém constante ao longo do tempo (equilíbrio dinâmico), não havendo acúmulo no sistema. Por último, não são avaliadas alterações de temperatura nas reações cinéticas, mantendo a mesma como um parâmetro constante no modelo, bem como não são consideradas perdas por evaporação. Com base nas simplificações adotadas, a equação resultante do modelo pode ser expressa por (6).

$0=\mathrm{W}_{\text {in }}-\mathrm{W}_{\text {out }}-\mathrm{u} \cdot \mathrm{V} \cdot \frac{\partial \mathrm{C}}{\partial \mathrm{x}}-\mathrm{k} \cdot \mathrm{C} \cdot \mathrm{V}$

O corpo hídrico foi dividido em segmentos ou volumes de controle $n$ (Figura 1), de modo que o equacionamento resulta em (7).

$Q_{n, n+1} \cdot C_{n}=Q_{n-1, n} \cdot C_{n-1}+W_{\text {in }}-W_{\text {out }}-k_{n} \cdot C_{n} \cdot V_{n}$

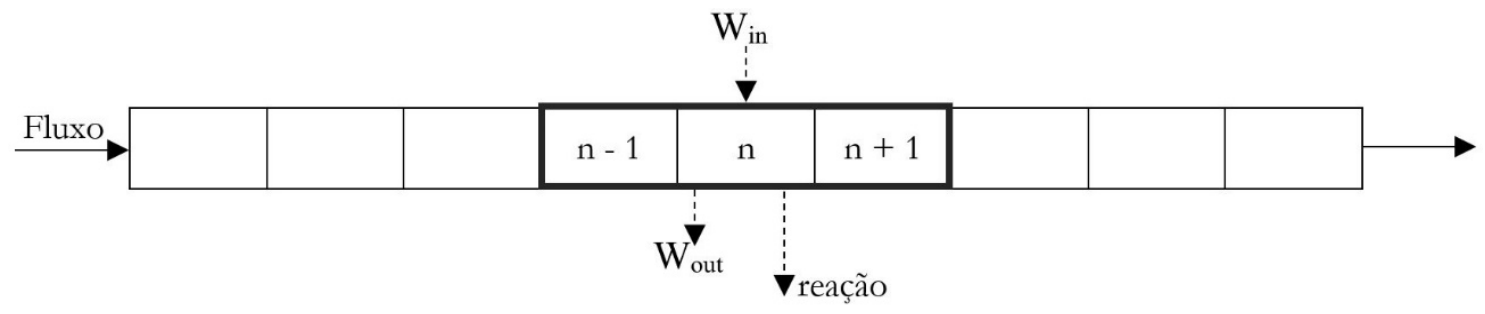

Figura 1: Representação de balanço de massa simplificado para um volume de controle. 
De maneira a possibilitar a inserção de cargas de diferentes usuários (exemplo: urbano, irrigante e industrial), estes são representados pelo índice $i$. As captações são discretizadas pela variável $s$, enquanto que os lançamentos são resultantes de uma fração de retorno ret. A carga externa $W_{n}$ representa a diferença resultante das captações e lançamentos dos diferentes usuários em cada trecho $n$, enquanto que $W_{\text {natural, }}$ representa a contribuição natural de cada sub-bacia para o trecho correspondente $n$. Para representar o processo de autodepuração do rio, as concentrações são corrigidas pelo fator $F$. 0 transporte de massa resultante é dado pelas equações 8 a 11.

$$
\begin{array}{lc}
\mathrm{Q}_{\mathrm{n}} \cdot \mathrm{C}_{\mathrm{n}}=\mathrm{Q}_{\mathrm{n}-1} \cdot \mathrm{C}_{\mathrm{n}-1} \cdot \mathrm{F}+\mathrm{W}_{\mathrm{n}}+\mathrm{W}_{\text {natural }_{\mathrm{n}}} & \forall \mathrm{n} \\
\mathrm{W}_{\text {natural }_{\mathrm{n}}}=\left(\mathrm{Q}_{\mathrm{p}_{\mathrm{n}}}-\mathrm{Q}_{\left.\mathrm{p}_{\mathrm{n}-1}\right) \cdot \mathrm{C}_{\text {natural }_{\mathrm{n}}}}\right. & \forall \mathrm{n} \\
\mathrm{W}_{\mathrm{n}}=\sum_{\mathrm{i}=1}^{\mathrm{I}} \mathrm{ret}_{\mathrm{i}, \mathrm{n}} \cdot \mathrm{s}_{\mathrm{i}, \mathrm{n}} \cdot \mathrm{D}_{\mathrm{i}} \cdot \mathrm{F}-\sum_{\mathrm{i}=1}^{\mathrm{I}} \mathrm{s}_{\mathrm{i}, \mathrm{n}} \cdot \mathrm{C}_{\mathrm{n}-1} \cdot \mathrm{F} & \forall \mathrm{i}, \mathrm{n} \\
\mathrm{F}=\mathrm{e}^{-\mathrm{k} \cdot \mathrm{t}_{\text {traveln }} \cdot \mathrm{LP}} &
\end{array}
$$

onde:

Índice $n$ representa a divisão do rio em trechos $(n=1,2, \ldots, N)$

$\mathrm{Q}_{\mathrm{n}}$ é a vazão resultante no final de cada trecho $n\left[\mathrm{M}^{3} \mathrm{~T}^{-1}\right]$

$\mathrm{C}_{\mathrm{n}}$ é a concentração do parâmetro de qualidade no final de cada trecho $n\left[\mathrm{M} \mathrm{L}^{-3}\right]$

$\mathrm{W}_{\mathrm{n}}$ é a carga total do parâmetro de qualidade resultante de captações e lançamento no trecho $n\left[\mathrm{M} \mathrm{T}^{-1}\right]$

$\mathrm{W}_{\text {natural }}{ }_{n}$ é a carga natural do parâmetro no trecho $n$, contribuição de cada sub-bacia para cada trecho [M T-1]

$\mathrm{Q}_{\mathrm{p}_{\mathrm{n}}}$ é a vazão com probabilidade de excedência $p$ no final do trecho $n\left[\mathrm{M}^{3} \mathrm{~T}^{-1}\right]$

$\mathrm{C}_{\text {natural }}{ }_{n}$ é a concentração natural do parâmetro de qualidade no trecho $n\left[\mathrm{M} \mathrm{L}^{-3}\right]$

ret $_{\mathrm{i}, \mathrm{n}}$ é a fração decimal de retorno (lançamento) do usuário $i$ no trecho $n$

$\mathrm{s}_{\mathrm{i}, \mathrm{n}}$ é a vazão captada pelo usuário $i$ no trecho $n\left[\mathrm{M}^{3} \mathrm{~T}^{-1}\right]$

$\mathrm{D}_{\mathrm{i}}$ é a concentração da substância no lançamento pelo usuário $i\left[\mathrm{M} \mathrm{L}^{-3}\right]$

F é o fator de depuração

LP é a fração decimal que representa a posição do lançamento dentro do trecho $n$. Para calcular a depuração da carga resultante do transporte entre os trechos $n$-1 e $n$ através da equação 8, o LP deve ser igual a 1.

\section{APLICAÇÃo}

\section{1 Área de Estudo}

A Bacia Hidrográfica do Rio dos Sinos localiza-se na porção leste do Estado do Rio Grande do Sul (Figura 2). O Rio dos Sinos deságua no Delta do Rio Jacuí, onde também afluem os Rios Caí e Gravataí. A área da Bacia é de $3.696 \mathrm{~km}^{2}$, o que corresponde aproximadamente a 4,4\% da área da Região Hidrográfica do Guaíba e a 1,3\% da área do Estado do Rio Grande do Sul. Ao todo, 32 municípios estão localizados total ou parcialmente na bacia, abrigando uma população estimada em 1.350.000 habitantes. As principais demandas de água na bacia são para abastecimento urbano (35\%), irrigação de arroz (53\%) e usos industriais (11\%), destacando, nesta categoria, as indústrias alimentícias, processamento de couro, metalúrgicas e refinaria de petróleo como principais usuárias de água (Profill, 2013).

Em estudo realizado por Profill (2013), a bacia do Sinos foi dividida em três grandes compartimentos (Alto, Médio e Baixo) em que se destacam condições relativamente homogêneas de relevo e uso do solo. O Alto Sinos é delimitado desde as nascentes, a montante da sede urbana de Caraá, até o rio da Ilha. 0 Médio Sinos é formado essencialmente pelo segmento correspondente à Bacia do Rio Paranhana e contribuintes menores nas margens esquerda e direita. Finalmente, o compartimento do Baixo Sinos, que pode ser lançado a partir da região de Sapiranga e Campo Bom até foz, é onde estão localizadas as sedes urbanas das maiores cidades da Bacia (Novo Hamburgo, São Leopoldo, Esteio, Sapucaia do Sul e Canoas). A divisão hidrográfica em alto, médio e baixo sinos também é considerada pelo Plano de Bacia (Profill, 2013) como base para a discretização em unidades de estudo (sub-bacias) e realização dos cálculos de disponibilidade hídrica e demandas. 
O desenvolvimento urbano e industrial ocorrido nas últimas décadas, não acompanhado por investimentos compatíveis de controle de poluição, resultaram em problemas críticos de poluição. O Rio dos Sinos é posicionado como o quarto mais poluído do Brasil (IBGE, 2010), sendo que apenas $4,5 \%$ população urbana total da bacia conta com sistema de coleta e tratamento de esgotos (Concremat, 2014).

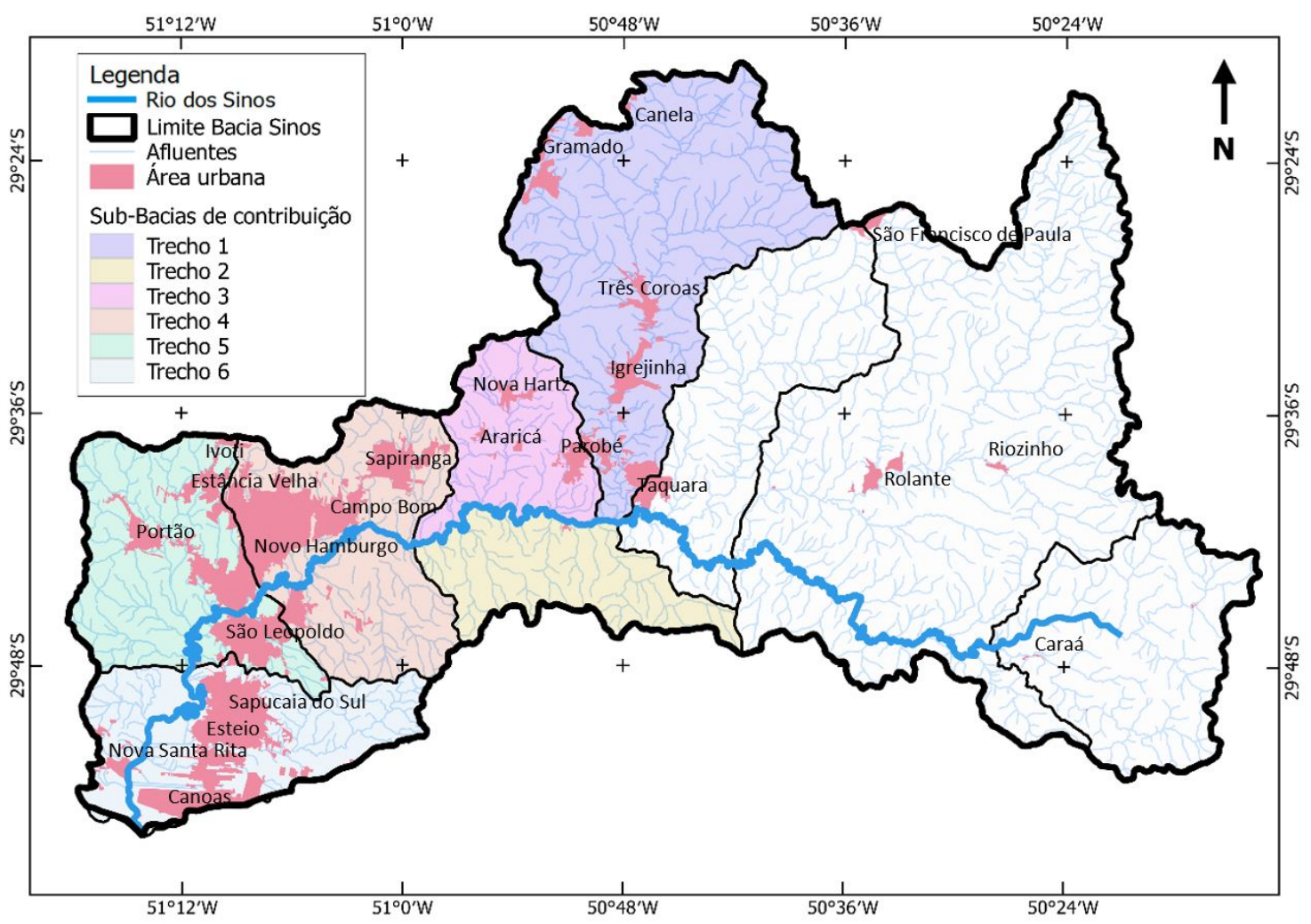

Figura 2: Bacia hidrográfica do Rio dos Sinos e divisão em trechos.

\subsection{Cenário avaliado}

O cenário atual da qualidade da água do Rio dos Sinos em períodos críticos de baixa vazão foi simulado em ambos modelos (Modelo de Referência HEC-RAS e Modelo Simplificado) incorporando os lançamentos de efluentes domésticos dos municípios integrantes da bacia. Os parâmetros de qualidade da água avaliados foram DBO e Coliformes termotolerantes por serem representativos quanto à poluição doméstica. Não foram avaliados lançamentos industriais ou outros de ordem difusa.

\subsubsection{Dados de entrada}

Os trechos do Rio dos Sinos utilizados nas simulações englobam a divisão médio e baixo Sinos com uma extensão total de $108 \mathrm{~km}$. A escolha desta extensão deu-se pela disponibilidade de dados topobatimétricos (levantamento realizado como parte integrante dos estudos do Plano de Bacia do Rio do Sinos), bem como por ser mais representativa quanto às concentrações populacionais.

Para simular a condição mais restritiva de qualidade da água, as vazões adotadas na simulação foram as mínimas de permanência $Q_{90}$ para cada sub-bacia de acordo com estudo apresentado no diagnóstico do Plano de Bacia (Sinos, 2010). O estudo foi baseado na série de vazões diárias observadas para o período de 1965 a 2007 da estação Fluviométrica de Campo Bom (localizada no Rio dos Sinos a $67 \mathrm{~km}$ da foz) e transferidas espacialmente para as sub-bacias através do conceito de vazão específica e ponderador de precipitação. As sub-bacias possuem área superior a $50 \mathrm{~km}^{2}$, o que contribui para que erros inerentes à espacialização não sejam significativos, segundo Tucci (2002). Como condição de jusante ocasionada pela oscilação de nível no Delta do Jacuí e Lago Guaíba considerou-se o nível que poderia apresentar maior condição de restrição, nível baixo (Datum Imbituba), igual a -0,2 m (Profill, 2014). 
A qualidade da água do Rio dos Sinos foi avaliada com base nos dados da rede de monitoramento gerenciada pela Fundação Estadual de Proteção Ambiental do Rio Grande do Sul (FEPAM) e disponibilizados pela própria entidade. No total, dados de 9 pontos da rede de monitoramento foram utilizados, os quais estão distribuídos ao longo da calha na extensão de 8 e $96 \mathrm{~km} \mathrm{da} \mathrm{foz.}$ 0 monitoramento ocorre desde 1990 com operação mensal, porém trabalhou-se apenas com os dados de 2005 a 2015, por ser um período mais recente, com boa representatividade e que não apresenta grandes desvios populacionais que poderiam ocasionar variações acentuadas de carga orgânica na bacia.

Para avaliar os valores de concentração relacionados apenas a vazões mínimas, foram filtrados da série histórica de vazões (estação fluviométrica de Campo Bom) as datas cuja vazão fosse menor ou igual a Q90. Em seguida, esses valores foram pareados com os dados da série histórica de qualidade da água referente à mesma data de medição. No caso do parâmetro DBO, para o total dos 9 pontos de monitoramento, foram obtidos 135 valores de concentração referentes a vazões mínimas (igual ou menor a Q90) no período de 2005 a 2015 distribuídos da seguinte forma: ponto $8 \mathrm{~km}$ - 15 valores; ponto $28 \mathrm{~km}$ - 17 valores; ponto $36 \mathrm{~km}$-15 valores; ponto $38 \mathrm{~km}$ - 17 valores; ponto $44 \mathrm{~km}$ - 18 valores; ponto $48 \mathrm{~km}$ - 13 valores; ponto $56 \mathrm{~km}$ - 19 valores; ponto $66 \mathrm{~km}$ - 7 valores e ponto $96 \mathrm{~km}$ - 14 valores. Para cada ponto de monitoramento, calculou-se a média dos valores de concentração disponíveis. Quanto ao parâmetro Coliformes termotolerantes, devido ao número reduzido de dados da rede de monitoramento, decidiu-se utilizar a média das concentrações observadas da série total do período de 2005 a 2015 em cada ponto de monitoramento por melhor representar a tendência das concentrações ao longo do rio.

\subsubsection{Modelo de Referência HEC-RAS}

O HEC-RAS é um programa computacional gratuito que permite simular escoamentos em rios e canais em regime permanente e não permanente, bem como realizar análise de transporte de sedimentos e modelagem da qualidade da água. 0 mesmo foi utilizado para comparação e avaliação do modelo simplificado proposto. Entre os parâmetros de qualidade da água passíveis de simulação na versão 5.05 utilizada estão: algas, oxigênio dissolvido, demanda carbonácea de oxigênio dissolvido, ortofosfato dissolvido, fósforo orgânico dissolvido, nitrogênio amoniacal dissolvido, nitrito dissolvido, nitrato dissolvido e nitrogênio orgânico dissolvido. 0 programa ainda permite a modelagem de constituintes arbitrários através da inserção de parâmetros relacionados às taxas cinéticas de decaimento ou crescimento (USACE, 2016). Os parâmetros de qualidade da água são modelados pela equação de advecção e dispersão unidimensional, sendo o rio/canal dividido em células com pontos computacionais localizados no centro das mesmas. Fluxos entre cada um dos parâmetros de qualidade são controlados pelo usuário através do ajuste das taxas de decaimento/crescimento e outros fatores associados.

Por meio de imagem orbital LANDSAT 8, o perfil do rio dos sinos foi traçado no programa HEC-RAS, sendo, em seguida, inseridas as 23 seções transversais resultantes do levantamento topobatimétrico ao longo da calha menor do rio. As seções foram ainda interpoladas a uma distância de 50 m, a fim de melhor cobrir as variações do terreno. Os dados de vazão mínima $\left(\mathrm{Q}_{90}\right)$ foram distribuídos ao longo de sua extensão de acordo com a localização do exutório de cada sub-bacia correspondente do estudo em Profill (2013). A simulação hidráulica possibilitou encontrar os valores de velocidade em cada seção interpolada e assim obter o tempo de percurso entre as mesmas. Este tempo foi então posteriormente utilizado no modelo simplificado na equação de decaimento (11).

Para a simulação da qualidade da água decorrente das cargas domésticas de esgoto sanitário, os lançamentos foram calculados de acordo com a população residente na bacia (Profill, 2013) e distribuídos ao longo de cada trecho $n$, conforme posicionamento das manchas urbanas. Os municípios de Parobé, Araricá, Sapiranga e Campo Bom foram representados de forma pontual, enquanto que os demais (Novo Hamburgo, Estância Velha, São Leopoldo, Portão, Sapucaia do Sul, Esteio e Canoas) foram representados por lançamentos distribuídos (tabela 1). Foram considerados valores de captação de água de 200 L/hab.dia, geração de esgoto per capita de 160 L/hab.dia, concentração de DBO efluente de $312 \mathrm{mg} / \mathrm{L}$ e Coliformes termotolerantes $1.10^{7} \mathrm{NMP} / 100 \mathrm{~mL}$ (Von Sperling, 2007).

A temperatura da água foi considerada constante na modelagem $\left(20^{\circ} \mathrm{C}\right)$. As taxas decaimento de primeira ordem para o parâmetro DBO foram ajustadas manualmente na interface de entrada do programa, enquanto que coliformes termotolerantes foi modelado como um parâmetro não conservativo arbitrário com decaimento de primeira ordem. Como concentração de base da bacia dos parâmetros de qualidade avaliados (condição natural do rio sem lançamento de cargas), foram utilizadas as concentrações médias da estação de monitoramento SI096 da Fepam, a qual localiza-se 
próxima à cabeceira do comprimento simulado. Por fim, foi utilizada a função do programa que permite calcular automaticamente os coeficientes de dispersão com base nas variáveis hidráulicas de cada seção do canal, conforme especificado em USACE (2016).

Realizada a calibração do Modelo de Referência HEC-RAS, foram utilizados os valores de tempo de percurso de cada trecho $n$ e os parâmetros cinéticos (kDQo e kcol) resultantes para simular o mesmo cenário pelo Modelo Simplificado escrito em linguagem Matlab.

Tabela 1: Dados de entrada para modelagem em HEC-RAS.

\begin{tabular}{|c|c|c|c|}
\hline $\begin{array}{l}\text { Trechos (seções } \\
\text { topobatimétricas) }\end{array}$ & Distância da foz (km) & Vazão $\left(Q_{90}\right)\left(\mathrm{m}^{3} / \mathrm{s}\right)$ & Cidade Contribuinte \\
\hline 23 & 108 & 9,50 & \\
\hline 22 & 103 & 12,83 & \\
\hline 21 & 99 & 13,82 & Parobé(1) \\
\hline 20 & 90 & 14,22 & Araricá(1) \\
\hline 19 & 81 & 14,62 & \\
\hline 18 & 75 & 15,02 & \\
\hline 17 & 70 & 15,65 & Sapiranga(1) \\
\hline 16 & 63 & 16,13 & Campo Bom(1) \\
\hline 15 & 56 & 16,55 & \\
\hline 14 & 53 & 16,71 & $\begin{array}{l}\text { Novo Hamburgo(2) } \\
\text { Estância Velha(2) }\end{array}$ \\
\hline 13 & 47 & 16,87 & $\begin{array}{l}\text { Novo Hamburgo(2) }^{(2)} \\
\text { Estância Velha(2) }\end{array}$ \\
\hline 12 & 38 & 17,14 & $\begin{array}{c}\text { São Leopoldo(2) } \\
\text { Portão(2) } \\
\end{array}$ \\
\hline 11 & 33 & 17,82 & $\begin{array}{l}\text { São Leopoldo(2) } \\
\text { Portão(2) }\end{array}$ \\
\hline 10 & 26 & 18,49 & \\
\hline 09 & 21 & 18,72 & Sapucaia do Sul(2) \\
\hline 08 & 13 & 18,96 & $\begin{array}{c}\text { Sapucaia do Sul(2) } \\
\text { Esteio(2) } \\
\end{array}$ \\
\hline 07 & 10 & 19,19 & Esteio(2) \\
\hline 06 & 9 & 19,42 & Esteio(2) \\
\hline 05 & 7 & 19,62 & Esteio(2) \\
\hline 04 & 5 & 19,81 & Canoas(2) $^{2}$ \\
\hline 03 & 3 & 19,89 & Canoas(2) $^{2}$ \\
\hline 02 & 1 & 19,97 & \\
\hline 01 & 0 & 20,05 & \\
\hline
\end{tabular}

(1)lançamento pontual; (2)lançamento distribuído

\subsubsection{Modelo Simplificado}

Para configuração do Modelo Simplificado, o Rio do Sinos foi dividido em um total de 6 trechos (volumes de controle) com diferentes comprimentos, considerando a localização e divisão prévia das sub-bacias de contribuição em Profill (2013). Trechos $n=4$ a 6 pertencem à divisão principal Baixo Sinos e trechos $n=1$ a 3 pertencem à divisão Médio Sinos. Os volumes de controle (divisão de trechos $n$ ) adotados no modelo simplificado são maiores que os empregados por um modelo computacional, porém destaca-se que, uma vez validado para a divisão proposta, a discretização em mais trechos pode ser facilmente incorporada no modelo simplificado, conforme a disponibilidade de dados.

As cargas de esgoto doméstico situadas dentro de cada trecho $n$ foram somadas de forma a caracterizar um lançamento único e pontual. Seguindo a mesma lógica, as vazões dos tributários de cada trecho $\left(Q_{90}\right.$ das sub-bacias de contribuição) foram representadas como um único fluxo lateral ao final do trecho $n$ (tabela 2).

Por último, a fim de avaliar o peso que a parcela de transporte difusivo exerce na equação do transporte de massa, uma vez que a mesma foi desconsiderada no modelo simplificado, foram realizadas simulações com ambos modelos (Referência e Simplificado) inferindo que os parâmetros se comportam como substâncias conservativas (não sofrem depuração). Ou seja, em ambos os modelos, o coeficiente $k$ de decaimento foi igualado a zero. A modelagem da qualidade utilizou vazões 
mínimas $Q_{90}$, as quais, por consequência, resultam em velocidades baixas e proporcionam melhor base para análise das influências da difusão no transporte de massa.

Tabela 2: Dados de entrada para o Modelo Simplificado.

\begin{tabular}{|c|c|c|c|c|c|}
\hline $\begin{array}{l}\text { Divisão } \\
\text { Principal }\end{array}$ & Trechos & $\begin{array}{l}\text { Distância da foz } \\
(\mathbf{k m})\end{array}$ & $\begin{array}{c}\text { Vazão }\left(Q_{90}\right) \\
\text { final trecho } \\
\left(\mathrm{m}^{3} / \mathrm{s}\right)\end{array}$ & $\begin{array}{c}\text { Tempo de } \\
\text { percurso* travel } \\
\text { (dia) }\end{array}$ & Cidade contribuinte \\
\hline- & inicial & 108 & 9,50 & - & - \\
\hline \multirow{3}{*}{ Médio Sinos } & 1 & 97 & 13,90 & 0,30 & Parobé \\
\hline & 2 & 86 & 14,40 & 0,44 & - \\
\hline & 3 & 75 & 15,02 & 0,33 & Araricá \\
\hline \multirow{3}{*}{ Baixo Sinos } & 4 & 50 & 16,80 & 1,25 & $\begin{array}{c}\text { Sapiranga } \\
\text { Campo Bom }\end{array}$ \\
\hline & 5 & 25 & 18,53 & 1,85 & $\begin{array}{l}\text { Novo Hamburgo } \\
\text { Estância Velha } \\
\text { São Leopoldo } \\
\text { Portão }\end{array}$ \\
\hline & 6 & 0 & 20,05 & 5,36 & $\begin{array}{c}\text { Sapucaia do Sul } \\
\text { Esteio } \\
\text { Canoas } \\
\end{array}$ \\
\hline
\end{tabular}

* resultante da modelagem hidráulica em HEC-RAS; tempo para percorrer final do trecho n-1 até final do trecho n.

\subsubsection{Métricas de comparação}

Com o objetivo de testar a adequabilidade da simplificação para o caso proposto, o desempenho dos resultados obtidos foram avaliados aplicando o coeficiente de determinação $r^{2}$ (12) e Nash-Sutcliffe Efficiency NSE (13) (Krause et al., 2005; Waseem et al., 2017). 0 intervalo de $\mathrm{r}^{2}$ situa-se entre 0 e 1, sendo melhor o ajuste quanto mais próximo de 1 . Já para NSE, o intervalo situa-se entre 1 (ajuste perfeito) e $-\infty$.

$$
\begin{aligned}
& \mathrm{r}^{2}=\left(\frac{\sum_{\mathrm{n}=1}^{\mathrm{N}}\left(\mathrm{O}_{\mathrm{n}}-\overline{\mathrm{O}}\right)\left(\mathrm{P}_{\mathrm{n}}-\overline{\mathrm{P}}\right)}{\sqrt{\sum_{\mathrm{n}=1}^{\mathrm{N}}\left(\mathrm{O}_{\mathrm{n}}-\overline{\mathrm{O}}\right)^{2}} \sqrt{\sum_{\mathrm{n}=1}^{\mathrm{N}}\left(\mathrm{P}_{\mathrm{n}}-\overline{\mathrm{P}}\right)^{2}}}\right)^{2} \\
& \mathrm{NSE}=1-\frac{\sum_{\mathrm{n}=1}^{\mathrm{N}}\left(\mathrm{O}_{\mathrm{n}}-\mathrm{P}_{\mathrm{n}}\right)^{2}}{\sum_{\mathrm{n}=1}^{\mathrm{N}}\left(\mathrm{O}_{\mathrm{n}}-\overline{\mathrm{O}}\right)^{2}}
\end{aligned}
$$

onde: $\mathrm{O}_{\mathrm{n}}=$ Concentração observada no trecho $\mathrm{n} ; \mathrm{P}_{\mathrm{n}}=$ Concentração prevista no trecho $\mathrm{n}$; $\overline{\mathrm{O}}=$ média das concentrações observadas; $\overline{\mathrm{P}}=$ média das concentrações previstas.

Moriasi etal. (2015) propôs limites para uma classificação qualitativa dos índices estatísticos para vazão, sedimentos e nutrientes (fósforo e nitrogênio) (tabela 3). Apesar dos parâmetros DBO e Coliformes termotolerantes não estarem nesta classificação, os valores servem como base para comparação.

Tabela 3: Classificação critérios de desempenho.

\begin{tabular}{c|c|c|c}
\hline Parâmetro avaliado & NSE & \multicolumn{2}{c}{ Classificação } \\
\hline Vazão & NSE $>0,80$ & $\mathrm{R}^{2}>0,85$ & \multirow{2}{*}{ Muito bom } \\
\hline Sedimento/P & $\mathrm{NSE}>0,80$ & $\mathrm{R}^{2}>0,80$ & \\
\hline Nutriente (N) & $\mathrm{NSE}>0,65$ & $\mathrm{R}^{2}>0,70$ & \multirow{2}{*}{ Bom } \\
\hline Vazão & $0,70<\mathrm{NSE} \leq 0,80$ & $0,75<\mathrm{R}^{2} \leq 0,85$ & \multirow{2}{*}{ Satisfatório } \\
\hline Sedimento & $0,70<\mathrm{NSE} \leq 0,80$ & $0,65<\mathrm{R}^{2} \leq 0,80$ & \multirow{2}{*}{ Insatisfatório } \\
\hline Nutriente (N/P) & $0,50<\mathrm{NSE} \leq 0,65$ & $0,60<\mathrm{R}^{2} \leq 0,70$ & \\
\hline Vazão & $0,50<\mathrm{NSE} \leq 0,70$ & $0,60<\mathrm{R}^{2} \leq 0,75$ & \\
\hline Sedimento & $0,45<\mathrm{NSE} \leq 0,70$ & $0,40<\mathrm{R}^{2} \leq 0,65$ & \\
\hline Nutriente (N/P) & $0,35<\mathrm{NSE} \leq 0,50$ & $0,30<\mathrm{R}^{2} \leq 0,60$ & \\
\hline Vazão & $\mathrm{NSE} \leq 0,50$ & $\mathrm{R}^{2} \leq 0,60$ & \\
\hline Sedimento & $\mathrm{NSE} \leq 0,50$ & $\mathrm{R}^{2} \leq 0,40$ & \\
\hline Nutriente (N/P) & $\mathrm{NSE} \leq 0,35$ & $\mathrm{R}^{2} \leq 0,30$ &
\end{tabular}




\section{RESULTADOS}

\subsection{Calibração do Modelo de Referência}

A figura 3 apresenta os resultados do ajuste do Modelo de Referência para os parâmetros DBO e Coliformes termotolerantes. 0 modelo conseguiu reproduzir satisfatoriamente os principais comportamentos das concentrações observadas nos pontos de monitoramento, sendo os maiores incrementos observados entre os quilômetros 30 e 50 do rio, devido aos lançamentos das cidades de Novo Hamburgo, Estância Velha, São Leopoldo e Portão. No trecho inferior (30 km até a foz), mesmo havendo contribuições significativas de cargas domésticas das cidades de Sapucaia do Sul, Esteio e Canoas, observou-se um decaimento acentuado das concentrações. Devido ao remanso do Delta do Jacuí, há uma redução da velocidade e consequente aumento do tempo de percurso, o que pode influenciar a ocorrência deste decaimento. Comportamento semelhante também foi obtido na simulação da qualidade da água realizada no Plano de Bacia (Profill, 2014).
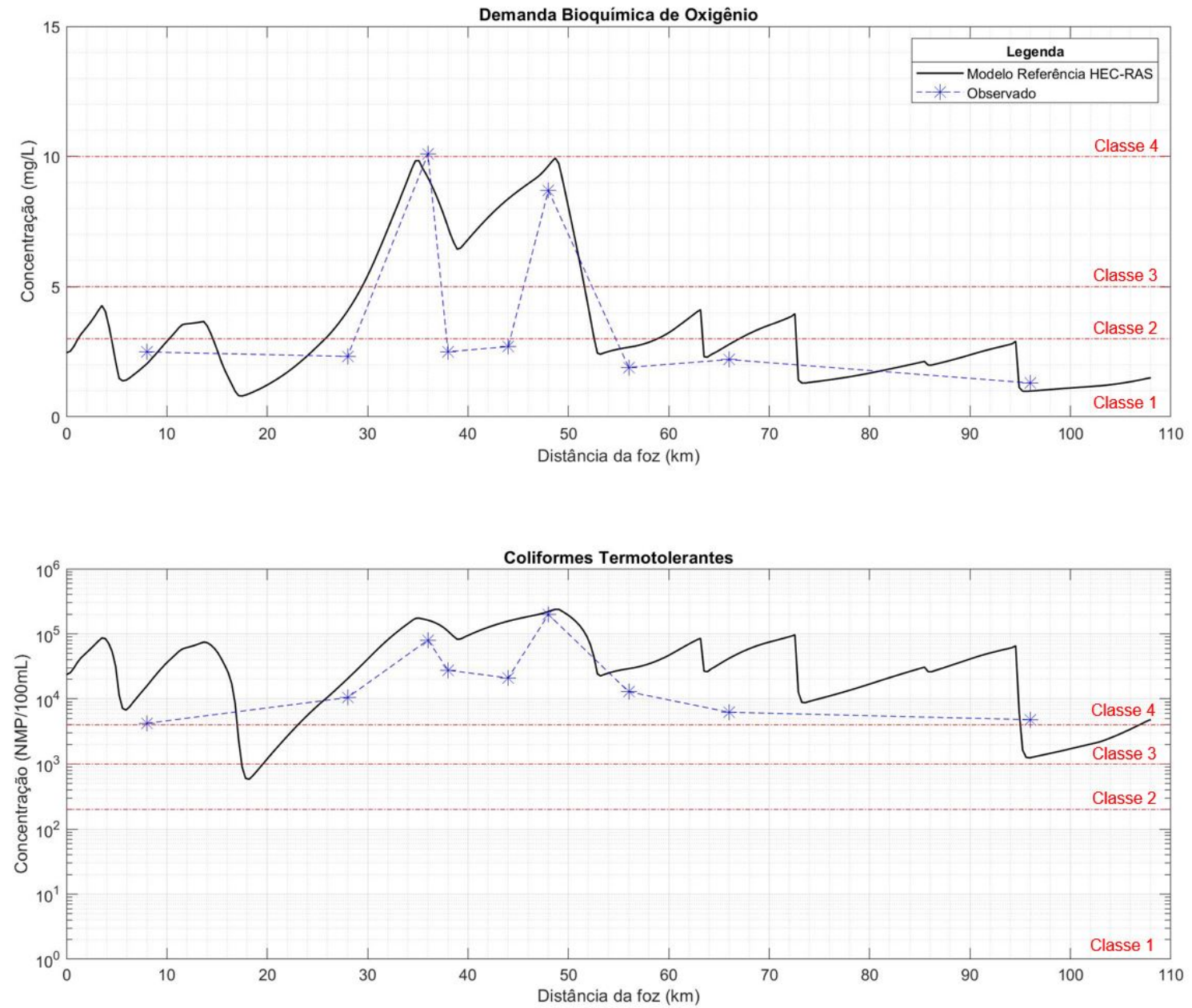

Figura 3: Resultado do ajuste do Modelo de Referência HEC-RAS com as médias das respectivas concentrações observadas da rede de monitoramento.

0 valor do coeficiente de decaimento ( $\mathrm{kBB}$ ) para DBO obtido na calibração foi de $1,2 \mathrm{~d}^{-1}$, sendo $0,7 \mathrm{~d}^{-1}$ referente à taxa de decomposição $\left(\mathrm{k}_{\mathrm{d}}\right)$ e $0,5 \mathrm{~d}^{-1}$ referente ao termo de remoção por sedimentação $\left(\mathrm{k}_{\mathrm{s}}\right)$. Ambos se apresentam dentro da faixa de valores citados pela literatura. 0 coeficiente de decaimento para coliformes termotolerantes ( $k_{\text {col }}$ ) obtido pela calibração foi de $2 \mathrm{~d}^{-1}$, também em acordo com a faixa de variações apresentada pela literatura.

Para o parâmetro DBO, os coeficientes $\mathrm{r}^{2}$ e NSE resultaram nos valores de 0,61 e 0,34, enquanto para Coliformes Termotolerantes os valores foram 0,65 e -0,15 respectivamente. Os resultados apontam valores satisfatórios para o coeficiente $\mathrm{r}^{2}$ e insatisfatório para NSE. Apesar do modelo HEC-RAS produzir uma série de pontos ao longo do trecho modelado, apenas 9 deles foram utilizados para a comparação 
por representarem a mesma posição no espaço dos pontos observados. 0 reduzido número de pontos pode ter influenciado o resultado de desempenho negativamente, uma vez que um ponto ruim passa a ter grande sensibilidade no resultado final, como é o caso do coeficiente NSE.

Após atingir um pico de concentração no $\mathrm{km} \mathrm{44}$, houve uma redução da concentração influenciada pelo decaimento, porém esta redução não foi o suficiente para atingir as concentrações observadas nos km 35 e 42. Neste caso, além do decaimento, outros fatores podem estar influenciando a redução da concentração entre estes dois trechos. Uma avaliação mais detalhada está fora do escopo deste estudo, uma vez que o objetivo é comparar os resultados entre modelos.

Os gráficos da figura 3 ainda possibilitam avaliar as variações das concentrações, conforme a classificação utilizada pela CONAMA 357/2005. Para o parâmetro DBO, as variações de concentrações conseguem acompanhar todas as classes (1 a 4), enquanto para o parâmetro coliformes termotolerantes, as variações ocorrem dentro da mesma classe (classe 4).

\subsection{Avaliação da parcela difusiva do transporte de massa}

Através da figura 4 é possível verificar que a parcela difusiva exerce baixa influência para o caso estudado. O Modelo Simplificado, o qual não apresenta a parcela difusiva no equacionamento, consegue acompanhar a tendência de aumento na concentração dos parâmetros avaliados, DBO e coliformes termotolerantes, ao longo de sua extensão da nascente à foz (aqui avaliados de forma conservativa) de forma aproximada com o resultado do Modelo de Referência. As principais variações observadas entre modelos são decorrentes da simplificação quanto ao somatório das cargas e lançamentos serem consideradas em um único ponto dentro de cada trecho. 0 valor do coeficiente $\mathrm{r}^{2}$ e NSE resultante da comparação entre modelos para a mesma distância de trecho é de 0,93 e 0,92 respectivamente para ambos parâmetros de qualidade, o que indica uma boa correlação.
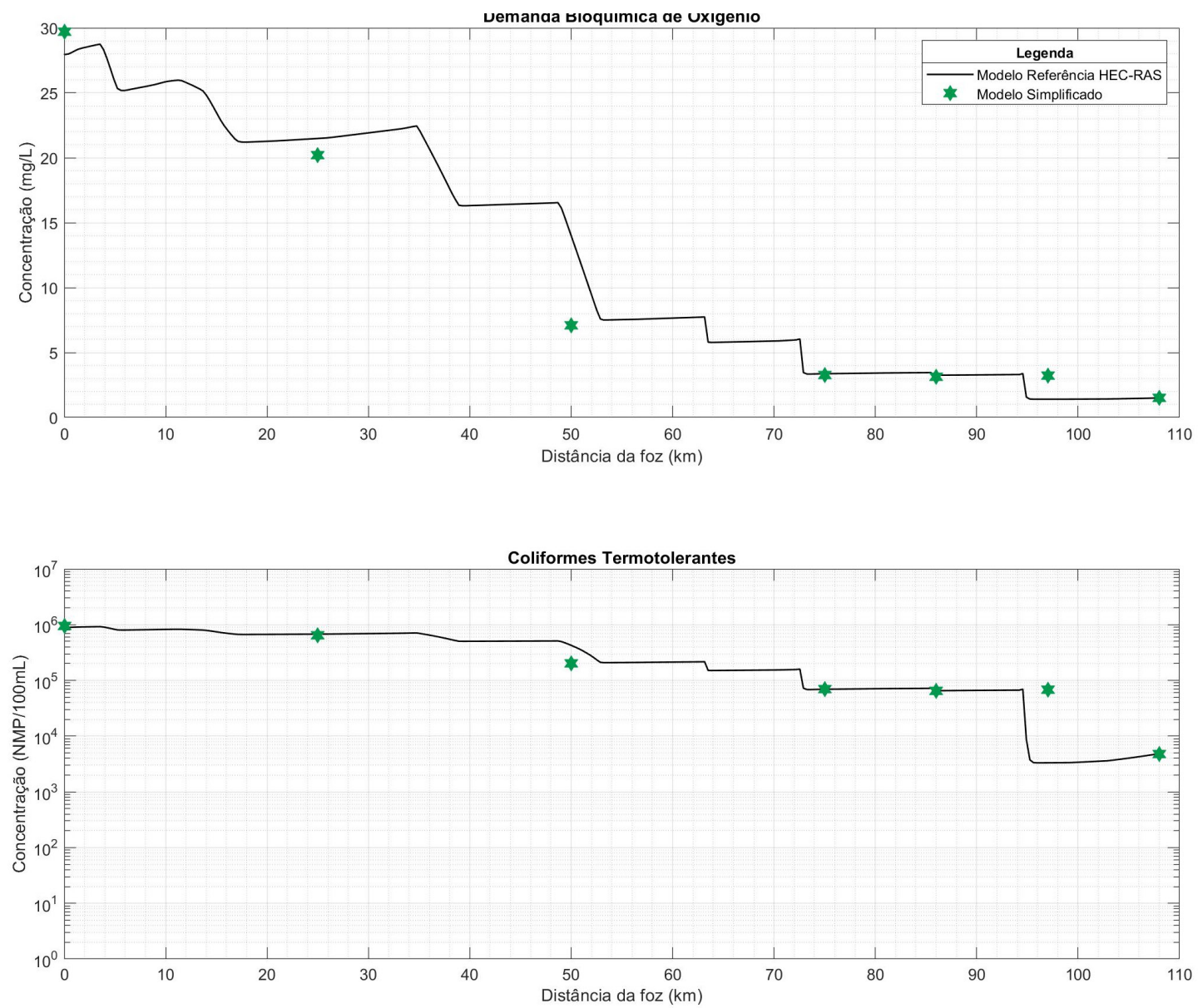

Figura 4: Avaliação do comportamento considerando os parâmetros de forma conservativa, Modelo de Referência e Modelo Simplificado. 


\subsection{Comparação das simulações de qualidade da água entre modelos (Simplificado e de Referência)}

A figura 5 apresenta a comparação dos resultados das simulações do Modelo de Referência e Modelo Simplificado para o mesmo cenário. 0 Modelo Simplificado consegue acompanhar o aumento e variações de concentrações ao longo de sua extensão de maneira bastante condizente, porém com deslocamento no espaço (extensão do rio), principalmente pelo motivo do modelo simplificado considerar o somatório das cargas de cada trecho lançadas em um único ponto. 0 deslocamento entre os picos, principalmente o situado a $25 \mathrm{~km}$ da foz, resultou em baixo desempenho para ambos parâmetros de qualidade ( $\mathrm{r}^{2}$ igual a 0,05 e NSE igual a -0,26).

Por outro lado, ao utilizar as distâncias em que as mesmas concentrações ocorrem entre modelos (como exemplo, a concentração de 9,7 mg/L de DBO ocorre no km 25 para o Modelo Simplificado e no km 35 para o Modelo de Referência), o coeficiente $\mathrm{r}^{2}$ resulta em 0,93 e NSE em 0,91 para o parâmetro DBO, enquanto que para o parâmetro coliformes termotolerantes o valor fica em 0,98 e 0,96 respectivamente. A diferença de $10 \mathrm{~km}$ é inferior ao comprimento total do trecho igual a $25 \mathrm{~km}$ adotado no Modelo Simplificado. Isso demonstra que, apesar do deslocamento no espaço, o resultando se encontra dentro do comprimento total do trecho avaliado. Caso o resultado da concentração seja mais importante que o posicionamento da mesma ao longo do rio, a divisão em 6 trechos proposta é o suficiente. Todavia, se for desejado aumentar o ajuste entre a concentração e o seu posicionamento no espaço, sugere-se aumentar a divisão do rio em mais trechos, a fim de que as cargas dos lançamentos sejam melhor posicionadas. Este último está fora do escopo deste estudo, mas é sugerido para futuros trabalhos.
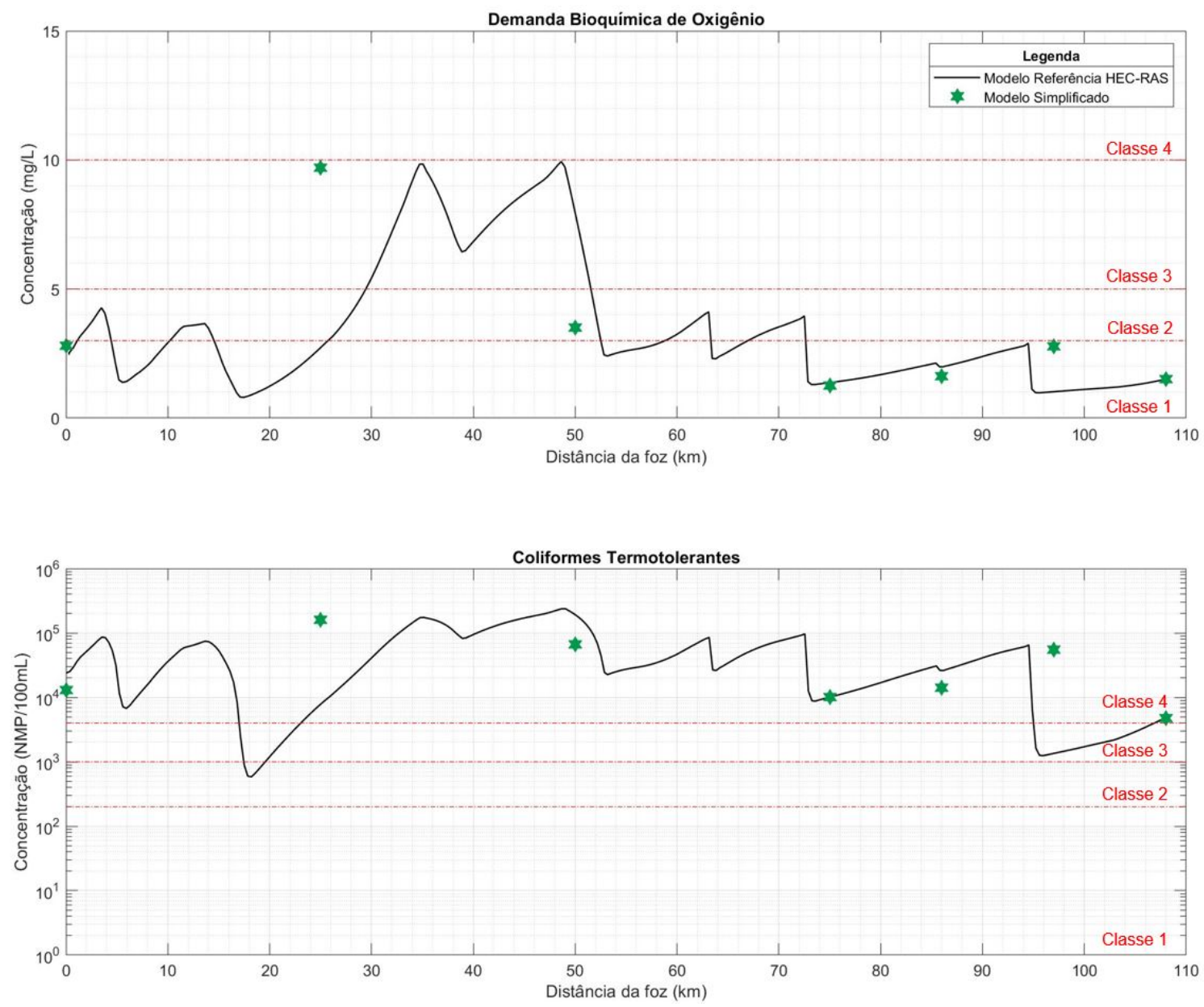

Figura 5: Comparação entre os resultados do parâmetro DBO e coliformes termotolerantes obtidos pelo Modelo de Referência HEC-RAS e o Modelo Simplificado. 


\section{INTEGRAÇÃO DO MODELO SIMPLIFICADO A MODELO HIDROECONÔMICO}

O modelo simplificado de qualidade da água foi utilizado em Dalcin (2019) como parte integrante da formulação do modelo hidroeconômico VISTA (Value Integrated Space-Temporal Allocation). 0 modelo apresenta três módulos de programação que operam de forma integrada, tendo por objetivo identificar diretrizes quanto à alocação de água (outorga), qualidade dos recursos hídricos (enquadramento) e emprego de instrumentos econômicos em uma bacia hidrográfica. A fim de avaliar as implicações das outorgas na qualidade da água, o módulo III do programa utiliza um algoritmo de programação não linear responsável pela distribuição espacial otimizada de outorgas na bacia hidrográfica para diferentes usuários sujeito a restrições de qualidade da água dadas pelo enquadramento. Dentro do processo de simulação da qualidade da água deste módulo, a variável $s_{i, n}$ da equação 10 se apresenta como uma variável de otimização, a qual segue função objetivo. Dessa forma, o algoritmo permite trabalhar com o processo de otimização e simulação de forma simultânea, bem como em conjunto com outros algoritmos de programação dinâmica e multiobjetivo que fazem parte do modelo.

\section{CONCLUSÃO E RECOMENDAÇÕES}

O presente artigo apresentou a base para formulação e simplificação de um modelo de qualidade da água para o Rio dos Sinos. A adequabilidade do Modelo Simplificado foi testada através da avaliação do mesmo cenário pelo programa computacional de modelagem hidrodinâmica HEC-RAS (Modelo de Referência), previamente calibrado a partir de dados de topobatimetria, imagens de satélite e qualidade da água da rede de monitoramento do Rio dos Sinos. Os resultados mostraram-se satisfatórios quanto às simplificações consideradas. A parcela responsável pelo transporte difusivo de massa apresentou baixa influência no resultado final, mesmo utilizando vazões mínimas, como é o caso da $Q_{90}$ adotada neste estudo.

O Modelo Simplificado conseguiu acompanhar as variações de concentração ao longo da extensão do Rio dos Sinos, porém com deslocamento da sua posição espacial (comprimento do rio). A discretização em mais trechos pode ser facilmente incorporada no Modelo Simplificado de maneira a melhorar a distribuição espacial. Por fim, destaca-se que o modelo, dentro de suas limitações, é de fácil aplicação em rotinas de programação diversas, o que facilita o seu emprego em modelos integrados no planejamento e gestão de recursos hídricos em que são utilizadas técnicas de otimização e simulação de forma simultânea.

\section{AGRADECIMENTOS}

Os autores agradecem o apoio da CAPES no desenvolvimento deste trabalho através da concessão de bolsa de pesquisa.

\section{REFERÊNCIAS}

Amorim Filho, C. A. G. (2013). Alocação Econômica Ótima na Bacia do Rio Tapacurá-Pernambuco sob Diferentes Cenários Climáticos. Universidade Federal de Pernambuco.

Azevedo, B. L. G. T., Gates, T. K., Fontane, D. G., Labadie, J. W., \& Porto, R. L. (2000). Integration of water quantity and quality in strategic river basin planning. Water Resources Planning and Management, 126(April), 85-97.

Bandeira, A. D. P. F., \& Mendes, C. A. B. (2009). Simulação de um modelo econômico para redução de cargas de poluentes, decorrentes da atividade de mineração em bacias hidrográficas. Rega, 6(2), 5-18.

Bouhlel, M. A., Hwang, J. T., Bartoli, N., Lafage, R., Morlier, J., \& Martins, J. R. R. A. (2019). A Python surrogate modeling framework with derivatives. Advances in Engineering Software, March, 1-27. http://dx.doi.org/10.1016/j.advengsoft.2019.03.005.

Cai, X., McKinney, D. C., \& Lasdon, L. S. (2003). Integrated hydrologic-agronomic-economic model for river basin management. Journal of Water Resources Planning and Management, 129(1), 4-17 ST-Integrated hydrologicagronomic-economi. Recuperado em 25 de agosto de 2019, de internal-pdf://ximint030348905472/ximint03.pdf LB - ximint03

Chapra, S. C. (2008). Surface water-quality modeling (2nd ed.). Illinois: Waveland Press.

Chin, D. A. (2013). Water-quality engineering in natural systems: fate and transport processes in the water environment (2nd ed.). New Jersy: John Wiley and Sons. 
Concremat. (2014). Plano regional de saneamento básico: relatório do diagnóstico. Porto Alegre: Prosinos.

Dalcin, A. P. (2019). Proposta de integração de instrumentos de gestão para estratégias de alocação da água de longo prazo (Dissertação de mestrado). Universidade Federal do Rio Grande do Sul.

Davidsen, C., Liu, S., Mo, X., Holm, P. E., Trapp, S., Rosbjerg, D., \& Bauer-Gottwein, P. (2015). Hydroeconomic optimization of reservoir management under downstream water quality constraints. Journal of Hydrology (Amsterdam), 529(1), 1679-1689. http://dx.doi.org/10.1016/j.jhydrol.2015.08.018.

IBGE. (2010). Indicadores de desenvolvimento sustentável: Brasil 2010. Rio de Janeiro: IBGE.

Ji, Z.-G. (2017). Hydrodynamics and Water Quality. Hydrodynamics and Water Quality: Modeling Rivers, Lakes, and Estuaries. Hoboken, NJ, USA: John Wiley \& Sons, Inc. https://doi.org/10.1002/9781119371946.

Krause, P., Boyle, D. P., \& Bäse, F. (2005). Comparison of different efficiency criteria for hydrological model assessment. Advances in Geosciences, 5, 89-97. https://doi.org/10.5194/adgeo-5-89-2005.

Loucks, D. P., \& van Beek, E. (2017). Water Resource Systems Planning and Management. Cham: Springer International Publishing. https://doi.org/10.1007/978-3-319-44234-1.

Martin, J. L., \& McCutcheon, S. C. (1999). Hydrodynamics and transport for water quality modeling. CRC Press.

Mckinney, D. C., Cai, X., Rosegrant, M. W., Ringler, C., \& Scott, C. A. (1999). Modeling Water Resources Management at the Basin Level: Review and Future Directions. Water Management (SWIM Paper). Sri Lanka: Inernational Water Management Institute (IWMI). Recuperado em 25 de agosto de 2019, de http://www.iwmi.cgiar.org/Publications/SWIM_Papers/PDFs/SWIM06.PDF

Molinos-Senante, M., Hernández-Sancho, F., Mocholí-Arce, M., \& Sala-Garrido, R. (2014). A management and optimisation model for water supply planning in water deficit areas. Journal of Hydrology (Amsterdam), 515, 139-146. http://dx.doi.org/10.1016/j.jhydrol.2014.04.054.

Moraes, M. G. A., Amorim, C. A., Albuquerque Filho, B. E. M., \& Mendes, G. (2009). Avaliação de instrumentos econômicos alocativos na gestão de bacias hidrográficas usando modelo econômico-hidrológico integrado. REGA, 6(2), 49-64.

Moraes, M. M. G. A., Cai, X., Ringler, C., Albuquerque, B. E., Vieira da Rocha, S. P., \& Amorim, C. A. (2010). Joint water quantity-quality management in a biofuel production area: integrated economic-hydrologic modeling analysis. Journal of Water Resources Planning and Management, 136(4), 502-511. http://dx.doi.org/10.1061/(asce)wr.1943-5452.0000049.

Moraes, M., Cirilo, J., Sampaio, Y., \& Cai, X. (2008). Modelo econômico-hidrológico integrado para gestão de bacias hidrográficas: abordagem piece-by-piece para incorporar a capacidade de autodepuração dos rios e limites de trofia dos reservatórios. Revista Brasileira de Recursos Hídricos, 13(1), 43-55. Recuperado em 25 de agosto de 2019, de http://www.abrh.org.br/SGCv3/UserFiles/Sumarios/16c85f00878bf69ad4a3c94ae5d433f7_dffe8aeb0378 928b932d5acf589707ed.pdf

Moriasi, D. N., Gitau, M. W., Pai, N., \& Daggupati, P. (2015). Hydrologic and water quality models: performance measures and evaluation criteria. Transactions of the ASABE, 58(6), 1763-1785. http://dx.doi.org/10.13031/trans.58.10715.

Profill. (2013). Plano de Bacia do Rio dos Sinos: Relatório final RT1 Fase inicial e Fase A. Porto Alegre: Rio Grande do Sul. Recuperado em 25 de agosto de 2019, de http://www.comitesinos.com.br/planodebacia

Profill. (2014). Plano de Bacia do Rio dos Sinos: Relatório técnico 2 - RT2 Fase B - complementação do enquadramento. Porto Alegre: Profill engenharia e ambiente LTDA. Recuperado em 25 de agosto de 2019, de http://www.comitesinos.com.br/planodebacia

Qian, Z., Seepersad, C. C., Joseph, V. R., Allen, J. K., \& Wu, C. F. J. (2006). Building Surrogate Models Based on Detailed and Approximate Simulations. Journal of Mechanical Design, 128(4), 668. http://dx.doi.org/10.1115/1.2179459

Sinos. (2010). Plano de Gerenciamento da bacia hidrográfica do Rio dos Sinos: Atividade 3.3 - síntese da situação atual dos recursos hídricos. Porto Alegre.

Streeter, H. W., \& Phelps, E. B. (1925). A study of the pollution and natural purification of the Ohio River. Public Health Bulletin, 140.

Thomann, R. V., \& Mueller, J. A. (1987). Principles of surface water quality modeling and control. New York: Harper an Row Publishers.

Tucci, C. E. M. (2002). Regionalização de vazões (1st ed.). Porto Alegre: Ed. UFRGS.

USACE U. A. C. of Engineers. (2016). HEC-RAS river analysis system User's Manual. Davis, California. 
Von Sperling, M. (2007). Estudos e modelagem da qualidade da água de rios (1st ed.). Belo Horizonte: UFMG.

Wang, Y., Yang, J., \& Chang, J. (2019). Development of a coupled quantity-quality-environment water allocation model applying the optimization-simulation method. Journal of Cleaner Production, 213, 944-955. http://dx.doi.org/10.1016/j.jclepro.2018.12.065

Waseem, M., Mani, N., Andiego, G., \& Usman, M. (2017). A review of criteria of fit for hydrologycal models. International Research Journal of Engineering and Technologu, 4(11), 1765-1772.

Yang, X. S., Koziel, S., \& Leifsson, L. (2014). Computational optimization, modelling and simulation: Past, present and future. Procedia Computer Science, 29, 754-758. https://doi.org/10.1016/j.procs.2014.05.067.

\section{Contribuições dos autores}

Ana Paula Dalcin executou as atividades de construção científica do artigo, busca e interpretação de dados, simulações de qualidade da água e comparação e análise dos resultados.

Guilherme Fernandes Marques participou da construção científica do manuscrito, orientou quanto às atividades de busca e interpretação dos dados, comparação e análise dos resultados, bem como participou das etapas finais de leitura e revisão 